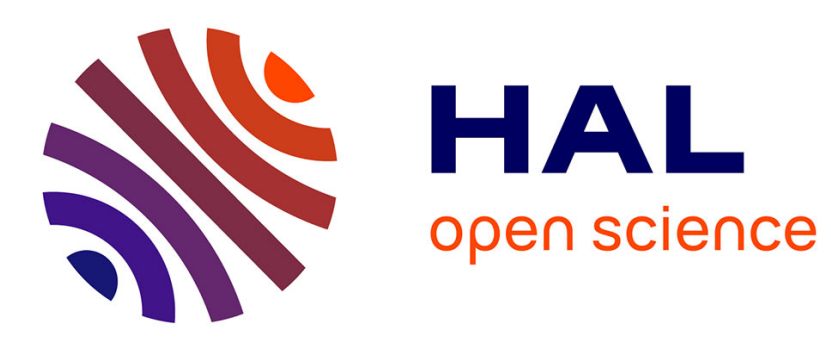

\title{
Robust compensation of a chattering time-varying input delay with jumps
}

Delphine Bresch-Pietri, Frédéric Mazenc, Nicolas Petit

\section{To cite this version:}

Delphine Bresch-Pietri, Frédéric Mazenc, Nicolas Petit. Robust compensation of a chattering time-varying input delay with jumps. Automatica, 2018, 92, pp.225-234. 10.1016/j.automatica.2018.03.058 . hal-01849024

\section{HAL Id: hal-01849024 \\ https://inria.hal.science/hal-01849024}

Submitted on 25 Jul 2018

HAL is a multi-disciplinary open access archive for the deposit and dissemination of scientific research documents, whether they are published or not. The documents may come from teaching and research institutions in France or abroad, or from public or private research centers.
L'archive ouverte pluridisciplinaire HAL, est destinée au dépôt et à la diffusion de documents scientifiques de niveau recherche, publiés ou non, émanant des établissements d'enseignement et de recherche français ou étrangers, des laboratoires publics ou privés. 


\title{
Robust compensation of a chattering time-varying input delay with jumps
}

\author{
Delphine Bresch-Pietri ${ }^{\mathrm{a}}$, Frederic Mazenc ${ }^{\mathrm{b}}$ and Nicolas Petit ${ }^{\mathrm{a}}$ \\ ${ }^{a}$ MINES ParisTech, PSL Research University, CAS - Centre Automatique et Systèmes, 60 bd St Michel 75006 Paris, FRANCE. \\ ${ }^{\mathrm{b}}$ EPI DISCO Inria-Saclay, L2S, CNRS CentraleSupelec, 3 rue Joliot Curie, 91192 Gif-sur-Yvette, FRANCE.
}

\begin{abstract}
We investigate the design of a prediction-based controller for a linear system subject to a time-varying input delay, not necessarily FirstIn/First-Out (FIFO). This means that the input signals can be reordered. The feedback law uses the current delay value in the prediction. It does not exactly compensate the delay in the closed-loop dynamics but does not require to predict future delay values, contrary to the conventional prediction technique. Modeling the input delay as a transport Partial Differential Equation, we prove asymptotic stabilization of the system state, that is, robust delay compensation, providing that the average $\mathscr{L}_{2}$-norm of the delay time-derivative over some time-window is sufficiently small and that the average time between two discontinuities (average dwell time) is sufficiently large.
\end{abstract}

Key words: delay systems; distributed parameter systems; reduction method; prediction-based control; time-varying delay; hybrid systems

\section{Introduction}

Time-delays are ubiquitous in engineering systems. They can take the form of communication lags or a physical deadtime and, in all cases, reveal troublesome in the design and tuning of feedback control laws. Delays are a central concern for numerous systems. When delay stems from transportation of material, as is observed in mixing plants for liquid or gaseous fluids [10] [29], automotive engine and exhaust line [12] or heat collector plant [31], the dead-time satisfies the First-In/First-Out (FIFO) principle by definition, i.e., the delay $D$ is such that $\dot{D}(t)<1$ for all time. However, this is not always the case. For example, communication delays can be subject to sudden variations and not satisfy the FIFO principle. This feature, sometimes refered to as fast-varying delay (see $[32,33]$ ), can also be exhibited for state- or inputdependent input delay systems [13], in which the delay variations can be related to the input in a very intricate manner, like, e.g., for crushing mill devices [30].

We investigate the design of a prediction-based control law [1] [20] [21] [34], which is state-of-the-art for constant input delay [8] [15] [17] [24] [26] [27] but has only been more recently used for time-varying delays (see [28]

Email addresses:

delphine.bresch-pietri@mines-paristech.fr (Delphine Bresch-Pietri), frederic.mazenc@l2s.centralesupelec.fr. (Frederic Mazenc), nicolas.petit@mines-paristech.fr (Nicolas Petit). or [18]). To compensate a varying input delay, the prediction has to be calculated over a time window the length of which matches the value of the future delay. In other words, one needs to predict the future variations of the delay to compensate it. This is the approach followed in [35] for a communication time-varying delay, the variations of which are provided by a given known model. It has also been used in $[2,3]$ for a state-dependent delay or in [4] for a delay depending on delayed state, where variations are anticipated by a careful prediction of the system state. However, in many cases, it is not possible to model the delay and, even if so, to predict the future delay values. For this reason, in this paper, in lieu of seeking exact delay compensation, we consider a prediction horizon equal to the current delay value, which is assumed to be known. This relaxed assumption is realistic. The delay itself can vary to a large extent, can be discontinuous and is not necessarily FIFO. By contrast with previous works accounting explicitly for the delay (that is, without recasting it as a disturbance) and assuming that $\dot{D}(t) \leq 1$ for $t \geq 0$ (see [5] [14] [36]), we allow the delay to be such that $\dot{D}(t)>1$ on some interval of time. A delay of this type, considered for the first time in preliminary study [9] in a prediction design context, is also considered in [25] and [23], but, in these papers the delay is supposed to be equal to a function of class $C^{1}$ plus a small discontinuous part, treated as a disturbance. We do not impose such an assumption; in other words, we consider delays with more general types of discontinuities, covering the case where they have large discontinuous jumps. 
We follow our preliminary study [9] which, as a first step, considered the delay function to be continuously differentiable, which is a demanding assumption from a practical point of view, and apply the novel time-varying version of Halany inequality proposed in [23] to address delay jumps. In this paper, as a result, the delay is only assumed to be piecewise continuously differentiable, encompassing potential sudden delay jumps and discontinuities, which are quite common, e.g., in the context of networks and communication protocols. Recasting the problem as an Ordinary Differential Equation (ODE) cascaded with a transport Partial Differential Equation (PDE), we use a backstepping transformation recently introduced in [19] to analyze the closed-loop stability. We prove asymptotic convergence of the system state providing that the delay time-derivative is sufficiently small in average, in the sense of an average $\mathscr{L}_{2}$-norm, and that the delay non-differentiability times are sufficiently sparse in time, in the sense of the average dwell time [16].

The paper is organized as follows. In Section 2, we introduce the problem at stake, before designing our control strategy and stating our main result. The latter is proven in Section 3. Section 4 presents an illustrative simulation example.

Notations. In the following, a function $f$ is said to be piecewise continuous on an interval $[a, b] \subset \mathbb{R}$ if the interval can be partitioned by a finite number of points $a=t_{0}<t_{1}<$ $\ldots<t_{n}=b$ so that $f$ is continuous on each subinterval $\left(t_{i-1}, t_{i}\right)$ and $f$ admits finite right-hand and left-hand limits at $t_{i}, i \in\{0, \ldots, n\}$. A function $f$ is said to be piecewise continuous on $\mathbb{R}$ if the restriction of $f$ to any interval is piecewise continuous. A function $f$ is said to be piecewise continuously differentiable on $\mathbb{R}$ if both $f$ and $f^{\prime}$ are piecewise continuous on $\mathbb{R}$. Standardly, we denote $\mathscr{C}_{p w}(I, \mathbb{R})\left(\operatorname{resp} . \mathscr{C}_{p w}(\mathbb{R}, \mathbb{R})\right)$ the set of real-valued piecewise continuous function on an interval $I \subset \mathbb{R}$ (resp. on $\mathbb{R}$ ) and $f\left(t^{+}\right)$(resp. $f\left(t^{-}\right)$) the righthand (resp. left-hand) limit of $f$ at point $t$, if it exists.

$|\cdot|$ is the usual Euclidean norm and, for a signal $u(x, \cdot)$ for $x \in[0,1],\|u(\cdot)\|$ denotes its spatial $\mathscr{L}_{2}$-norm, i.e.,

$$
\|u(t)\|=\sqrt{\int_{0}^{1} u(x, t)^{2} d x}
$$

In the sequel, integrals should be understood in the Riemann integrability sense, that is, when the signal $x \mapsto u(x, \cdot)$ is not defined on a set $S \subset[0,1]$ of measure zero, we write

$$
\|u(t)\|=\sqrt{\int_{0}^{1} u(x, t)^{2} d x}=\sqrt{\int_{[0,1] \backslash S} u(x, t)^{2} d x}
$$

and similarly for time signals. Finally, for a matrix $M$ the eigenvalues of which are all real numbers, $\underline{\lambda}(M)$ and $\bar{\lambda}(M)$ refer to the minimal and maximal eigenvalues of $M$.

\section{Problem statement and control design}

We consider the following (potentially) unstable linear dynamics

$$
\dot{X}(t)=A X(t)+B U(t-D(t))
$$

in which $X \in \mathbb{R}^{n}, U$ is scalar and the delay $D$ satisfies the following assumption.

Assumption 1 The delay $D$ is a piecewise continuously differentiable function with set of time instants of nondifferentiability

$$
\mathscr{T}=\left\{t_{i}, i \in \mathbb{N}\right\}
$$

and which satisfies

(i) $D(t) \in[\underline{D}, \bar{D}]$ for $t \geq 0$, with $0<\underline{D} \leq \bar{D}$

(ii) there exists $\underline{\Delta}>0$ such that $t_{i}-t_{j} \geq \underline{\Delta},\left(t_{j}, t_{i}\right) \in \mathscr{T}^{2}$, $i>j$

(iii) there exist $T>0$ and $\delta>0$ such that, for all $i \in \mathbb{N}$,

$$
\frac{1}{T} \int_{t}^{t+T} \dot{D}(s)^{2} d s \leq \delta, \quad t \in\left(t_{i}, t_{i+1}-T\right), \quad t_{i} \in \mathscr{T}
$$

Note that no assumption is made a priori on the timederivative of $D$. In particular, it is possible that $\dot{D}(t)>1$ for certain intervals of time. Also, it is worth observing that $D$ is not necessarily continuous at time $t_{i} \in \mathscr{T}$.

In the sequel, we consider that the current value of the delay is known.

Our control objective is to design a prediction-based controller stabilizing the plant (3), using the knowledge of the current value of the delay $D(t)$ at time $t \geq 0$. With this aim in view, consider the following control law

$$
U(t)=K\left[e^{A D(t)} X(t)+\int_{t-D(t)}^{t} e^{A(t-s)} B U(s) d s\right]
$$

in which the feedback gain $K$ is such that $A+B K$ is Hurwitz.

This controller approximately forecasts value of the state over a time window of varying length $D(t)$. Note that this controller does not exactly match the predicted system state on a time-horizon $D(t)$. Indeed, using the variation of constant formula

$$
\begin{aligned}
X(t+D(t))= & e^{A D(t)} X(t) \\
& +\int_{t-D(t)}^{t} e^{A(t-s)} B U(s+D(t)-D(s)) d s
\end{aligned}
$$


However, the integral in this last expression may not be implementable as it is not necessarily causal ${ }^{1}$. Further, even if one can implement this prediction, the involved integral can be approximated by the one used in (6) if $D(t)-D(s) \approx 0$ for "most" instants $t$, i.e., under the assumption that the variations of the delay are sufficiently small in average. As this assumption is the one which is required later in Theorem 1 to robustly compensate the delay, we rather use the prediction form (6) which is easier to implement but also, and, more importantly, always causal.

Of course, exact compensation of the delay is not achieved with this controller, as it is based on the (incorrect) assumption that $D\left(t^{\prime}\right)=D(t)$ for $t^{\prime}>t$. To do so, one would need to consider a time window of which length would exactly match the value of the future delay, as performed in [28] and [18]. In details, defining $\varphi(t)=t-D(t)$ and assuming that its inverse exists, exact delay-compensation is obtained with the feedback law $U(t)=K X\left(\varphi^{-1}(t)\right)$. Yet, implementing this relation requires to predict the future variations of the delay via $\varphi^{-1}(t)$. This may not be achieved in practice, when no delay model is available. More importantly, note that the inverse function $\varphi^{-1}(t)$ may not exist for all time, if $\dot{D}(s)>1$ for some instants as $\varphi$ may then be nonmonotonically increasing. This motivates our choice of the prediction-based controller (6).

Theorem 1 Consider the closed-loop system consisting of the dynamics (3) and the control law (6) in which the delay $D: \mathbb{R} \rightarrow[\underline{D}, \bar{D}]$ satisfies Assumption 1. Define the functional

$$
\Gamma(t)=|X(t)|^{2}+\int_{t-\bar{D}}^{t} U(s)^{2} d s
$$

and a chatter bound $N_{0}$ and an average dwell time $\tau_{D} \geq 0$ such that

$$
N_{D}(t, \tau) \leq N_{0}+\frac{t-\tau}{\tau_{D}}
$$

in which $N_{D}(t, \tau)$ denotes the number of discontinuities of $\dot{D}$ in the interval $(\tau, t)$. There exist $\delta^{*} \in(0,1)$ and $\tau_{D}^{*}>0$ such that, if $\delta<\delta^{*}$ and if $\tau_{D}>\tau_{D}^{*}$, then there exist two constants $\gamma, R>0$ such that

$$
\Gamma(t) \leq R \max _{s \in[-\bar{D}, 0]} \Gamma(s) e^{-\gamma t}, t \geq 0
$$

Equation (5) along with the condition $\delta<\delta^{*}$ allows the delay time-derivative to be quite large for some time instants. However, to guarantee stability, it requires it to be sufficiently small in average, in the sense of the average $\mathscr{L}_{2}$-norm given in (5). In particular, the delay function can be non-FIFO for some time instants, as long as it is most

\footnotetext{
1 In details, if there exists $s \in[t-D(t), t]$ such that $s-D(s) \geq$ $t-D(t)$, i.e., if the delay $D(t)$ is suddenly high and the information received at time $t$ older than some previously received) while the one employed in (6) always is.
}

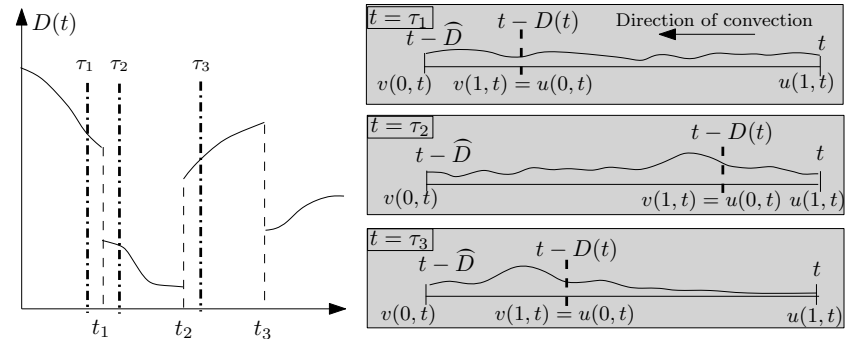

Fig. 1. Schematic views of the distributed variables introduced in (11)-(12) for delay variations pictured in the left plot.

of the time (i.e. as $\delta^{*}<1$ ). Second, delay jumps can occur provided that the average dwell time is large enough to guarantee stability.

Note that, as our prediction employs the current delay value $D(t)$ instead of the time horizon $\varphi^{-1}(t)$ to estimate the future system state, it can be highly inaccurate when the delay is fast varying. In this context, the requirement $\delta<\delta^{*}$ with $\delta$ introduced in (5) can also be interpreted as a condition for robust delay compensation achievement: if the delay varies sufficiently slowly most of the time, its current value $D(t)$ used for prediction will remain, sufficiently often, close enough to its future values for the corresponding prediction to guarantee closed-loop stabilization.

We now detail the proof of this theorem.

\section{Proof of Theorem 1}

\subsection{Reformulation of the plant as a cascade with a trans- port PDE}

As a first step in our analysis, we define $\widehat{D}>\bar{D}$ and introduce the two distributed actuators

$$
\begin{aligned}
& u(x, t)=U(t+D(t)(x-1)) \\
& v(x, t)=U(t-\widehat{D}+x(\widehat{D}-D(t)))
\end{aligned}
$$

to reformulate the plant (3) into a PDEs-ODE cascade. In details, the variable $u$ represents the history of the input on the (moving) horizon $[t-D(t), t]$ while $v$ completes it by the history of the input over the (moving) horizon $[t-\widehat{D}, t-D(t)]$. These variables are pictured in Figure 1.

Note that, from (6) and as the delay is piecewise continuously differentiable, the control law is also piecewise continuously differentiable and so are the distributed inputs $u$ and $v$ with respect to $x$ and t. To clarify this point, we first formulate the following intermediate results.

Lemma 1 The control law defined in (6) is continuously differentiable on the union of intervals $\mathbb{R} \backslash \mathscr{T}$. 
Proof Consider the Dini derivative of $U$

$$
\begin{aligned}
& \mathbb{D}^{+} U(t)=\limsup _{h \rightarrow 0^{+}} \frac{U(t+h)-U(t)}{h} \\
& =K\left[A\left[e^{A D(t)} X(t)+\int_{t-D(t)}^{t} e^{A(t-s)} B U(s) d s\right]\right. \\
& \left.\quad+B U(t)+\dot{D}(t) e^{A D(t)}[A X(t)+B U(t-D(t))]\right]
\end{aligned}
$$

One can observe that the right-hand term of this equation is well-defined and continuous as long as $t \notin \mathscr{T}$.

Lemma 2 For $t_{i} \in \mathscr{T}$, consider the sets

$$
\begin{aligned}
& \mathscr{D}_{i}=\left\{t \in \mathbb{R} \mid t \geq t_{i} \text { and } t-D(t) \leq t_{i}\right\} \\
& \tilde{\mathscr{D}}_{i}=\left\{t \in \mathbb{R} \mid t-D(t) \geq t_{i} \text { and } t-\widehat{D} \leq t_{i}\right\}
\end{aligned}
$$

and the variables

$$
\begin{aligned}
& x_{i}(t)=1+\frac{t_{i}-t}{D(t)}, \text { for } t \in \mathscr{D}_{i} \\
& \tilde{x}_{i}(t)=\frac{t_{i}+\widehat{D}-t}{\widehat{D}-D(t)}, \text { for } t \in \tilde{\mathscr{D}}_{i}
\end{aligned}
$$

Define

$$
\begin{aligned}
& \mathscr{X}(t)=\left\{x_{i}(t) \mid \mathscr{D}_{i} \ni t, i \in \mathbb{N}\right\} \\
& \tilde{X}(t)=\left\{\tilde{x}_{i}(t) \mid \tilde{\mathscr{D}}_{i} \ni t, i \in \mathbb{N}\right\}
\end{aligned}
$$

Then, the distributed variable $u$ (resp. v) is continuously differentiable on the set

$$
\begin{array}{r}
\mathscr{D}_{u}=\{(x, t) \mid t \notin \mathscr{T}, x \notin \mathscr{X}(t)\} \\
\text { resp. } \left.\mathscr{D}_{v}=\{(x, t) \mid t \notin \mathscr{T}, x \notin \mathscr{X}(t)\}\right)
\end{array}
$$

Further, the function $x_{i}$ (resp. $\tilde{x}_{i}$ ) is continuously differentiable for $t \in \mathscr{D}_{i} \backslash \mathscr{T}$ (resp. for $t \in \tilde{\mathscr{D}}_{i} \backslash \mathscr{T}$ ) and satisfies

$$
\begin{gathered}
1+\dot{D}(t)\left(x_{i}(t)-1\right)+D(t) \dot{x}_{i}(t)=0, t \in \mathscr{D}_{i} \backslash \mathscr{T} \\
\left(\text { resp. } 1+\dot{x}_{i}(t)(\widehat{D}-D(t))-x_{i}(t) \dot{D}(t)=0, t \in \tilde{\mathscr{D}}_{i} \backslash \mathscr{T}\right)
\end{gathered}
$$

Before, providing a proof of this lemma, it is useful to make a few comments on the definitions introduced above and illustrated on Fig. 2. First, $\widehat{D}>\bar{D}$ has been introduced only to guarantee the well-posedness of (17) (otherwise, for $\widehat{D}=$ $\bar{D}$, this variable could be undefined for some time instants such that $D(t)=\bar{D}$ ). Second, the subset of time $\mathscr{D}_{i}$ (resp. $\left.\tilde{\mathscr{D}}_{i}\right)$ is a union of intervals which gathers times for which a spatial non-differentiability point in $u$ (resp. v) related to the delay non-differentiability time $t_{i}$ exists. Conversely, for a given $t \in \mathbb{R}$, the space set $\mathscr{X}(t)$ (resp. $\tilde{X}(t))$ is a finite union of singletons which gathers, at a given time $t$, all existing points of spatial non-differentiability of $u$ (resp. $v$ ).

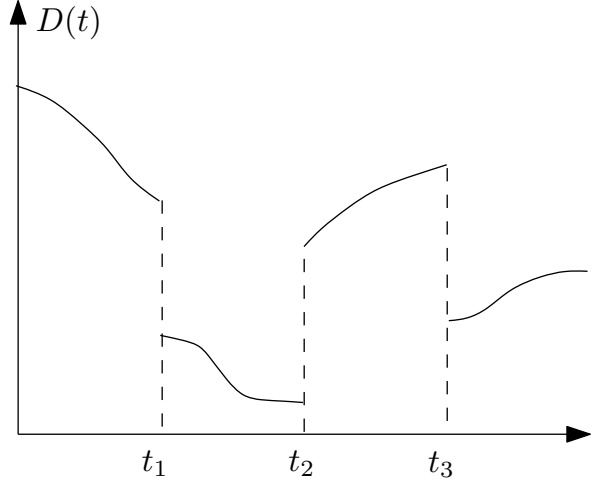

(a) Delay variations.

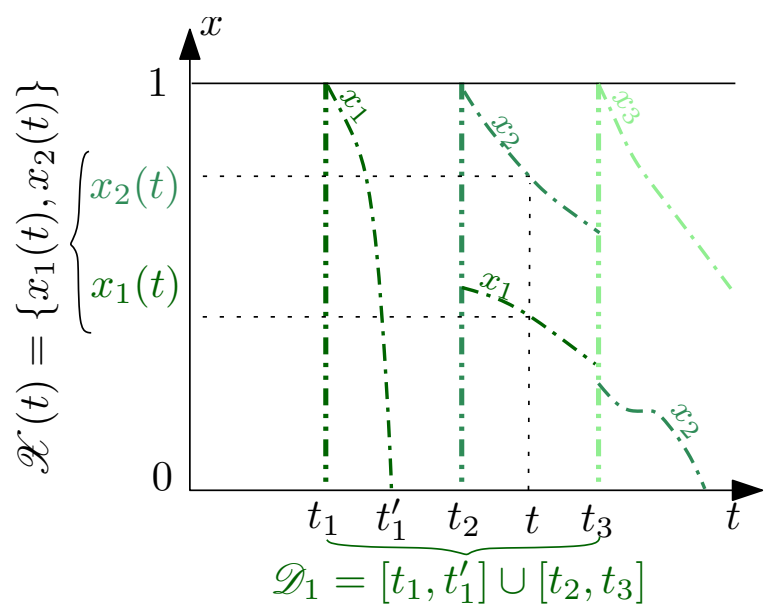

(b) Schematic view of the set of differentiability of $u$. Dasheddotted curves represent the sets where $u$ is not differentiable.

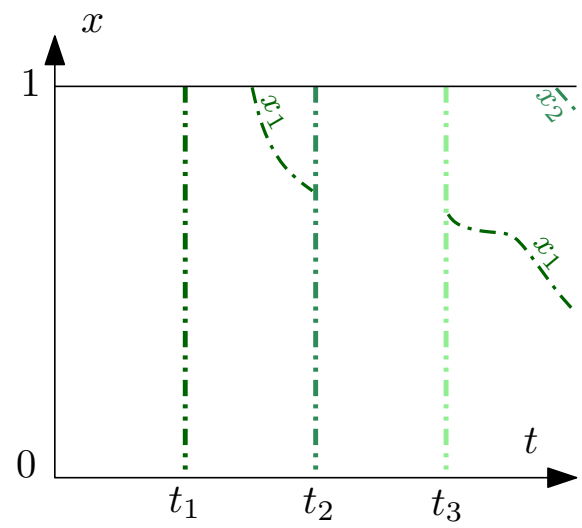

(c) Schematic view of the set of differentiability of $v$. Dashed-dotted curves represent the sets where $v$ is not differentiable. One can note that $\tilde{\mathscr{X}}(t)=\emptyset$ while $\mathscr{X}(t)=\left\{x_{1}(t), x_{2}(t)\right\}$.

Fig. 2. Schematic views of the sets introduced in Lemma 2 for a given delay function pictured in the left-hand plot 2(a).

In particular, this union is indeed finite because the delay non-differentiability times are separated by at least $\underline{\Delta}$ due to Assumption 1. 
Proof By definition, the time-derivative of the feedback $u$ defined in (11) is given as

$$
u_{t}(x, t)=(1+\dot{D}(t)(x-1)) \dot{U}(t+D(t)(x-1))
$$

It is straightforward to see the $u_{t}$ is not well-defined for $t \in \mathscr{T}$. Now, consider $t \notin \mathscr{T}$, then $u_{t}$ is well-defined provided that $x \in[0,1]$ is such that $t+D(t)(x-1) \notin \in \mathscr{T}$. This last condition can be formulated as $x \notin \mathscr{X}(t)$. Similar considerations can be made regarding the space-derivative of $u$. The result follows.

Finally, $x_{i}(t) \in \mathscr{X}(t)$ implies that $t+D(t)\left(x_{i}(t)-1\right)=t_{i}$. Taking a time-derivative of this expression, one obtains (22). Similar conclusions can be obtained for the distributed variable $v$ defined in (12) following the same steps, which concludes the proof.

This allows to rewrite the plant (3) as the following PDEsODE cascade

$$
\begin{aligned}
& \dot{X}(t)=A X(t)+B u(0, t) \\
& D(t) u_{t}=(1+\dot{D}(t)(x-1)) u_{x},(x, t) \in \mathscr{D}_{u} \\
& u(1, t)=U(t) \\
& u\left(x, t^{+}\right)=U\left(t^{+}+D\left(t^{+}\right)(x-1)\right), t \in \mathscr{T}, x \notin \mathscr{X}(t) \\
& (\widehat{D}-D(t)) v_{t}=(1-x \dot{D}(t)) v_{x},(x, t) \in \mathscr{D}_{v} \\
& v(1, t)=u(0, t) \\
& v\left(x, t^{+}\right)=U\left(t^{+}-\underline{D}+x\left(\widehat{D}-D\left(t^{+}\right)\right)\right), t \in \mathscr{T}, x \notin \mathscr{X}(t)
\end{aligned}
$$

This system is well-posed, in the sense of the following lemma, the proof of which is provided in Appendix.

Lemma 3 For any initial data $(u, v) \in L_{2}(0,1) \times L_{2}(0,1)$, the system (26)-(31) has a unique weak solution in $L_{2}(0,1) \times L_{2}(0,1)$.

In details, the input delay is now represented as the cascade of an ODE (25) fed by the output of a transport PDE (26)(28), with time- and space- varying propagation velocity, which can potentially be locally equal to zero or negative. This output also feeds a second transport PDE (29)-(31) with time- and space- varying propagation velocity. It is worth mentioning that one needs to take into account both distributed variables $(u, v)$ in the analysis to account for all potential values of the delay and the entire history of the input over the time interval $[t-\widehat{D}, t]$.

\subsection{Backstepping transformation and target system}

To analyse this closed-loop system, following [19], we define the backstepping transformation

$$
\begin{aligned}
w(x, t)= & u(x, t) \\
& -K\left[e^{A D(t) x} X(t)+D(t) \int_{0}^{x} e^{A D(t)(x-y)} B u(y, t) d y\right]
\end{aligned}
$$

As previously, before starting our analysis, we investigate the well-posedness of this distributed variable.

Lemma 4 The backstepping transformation defined in (32) is continuously differentiable for $(x, t) \in \mathscr{D}_{u}$, as introduced in (20).

Proof Taking a space-derivative of (32), one gets

$$
\begin{aligned}
w_{x}(x, t)= & u_{x}(x, t)-K\left[A D(t) e^{A D(t) x} X(t)+D(t) B u(x, t)\right. \\
& \left.+A D(t)^{2} \int_{0}^{x} e^{A D(t)(x-y)} B u(y, t) d y\right]
\end{aligned}
$$

which is well-defined for $(x, t) \in \mathscr{D}_{u}$, following Lemma 2 . Similarly, taking a time-derivative of (32), one gets

$$
\begin{aligned}
& w_{t}(x, t)=u_{t}(x, t) \\
& -K \dot{D}(t)\left[e^{A D(t) x} A x X(t)+\int_{0}^{x} e^{A D(t)(x-y)} B u(y, t) d y\right] \\
& -K\left[e^{A D(t) x}(A X+B u(0, t))+D(t) \frac{d}{d t}[\right. \\
& \left.\left.\sum_{\left(x_{i}, x_{i+1}\right) \in \mathscr{X}_{[0, x]}(t)^{2}, x_{i}<x_{i+1}} \int_{x_{i}^{+}}^{x_{i+1}^{-}} e^{A D(t)(x-y)} B u(y, t) d y\right]\right]
\end{aligned}
$$

in which we have introduced, for $(a, b) \in[0,1]^{2}$,

$$
\mathscr{X}_{[a, b]}(t)=\{a\} \cup\left\{x_{i} \in \mathscr{X}(t) \mid x_{i} \leq b\right\} \cup\{b\}
$$

and where

$$
\begin{aligned}
& \frac{d}{d t}\left[\sum_{\left(x_{i}, x_{i+1}\right) \in \mathscr{X}_{[0, x]}(t)^{2}, x_{i}<x_{i+1}} \int_{x_{i}^{-}}^{x_{i+1}^{+}} e^{A D(t)(x-y)} B u(y, t) d y\right] \\
& =\sum_{\left(x_{i}, x_{i+1}\right) \in \mathscr{X}_{[0, x]}(t)^{2}, x_{i}<x_{i+1}}\left[\int_{x_{i}^{+}}^{x_{i+1}^{-}} \frac{d}{d t}\left(e^{A D(t)(x-y)} B u(y, t)\right) d y\right. \\
& \left.+\dot{x}_{i+1} e^{A D(t)\left(x-x_{i+1}\right)} B u\left(x_{i+1}^{-}, t\right)-\dot{x}_{i} e^{A D(t)\left(x-x_{i}\right)} B u\left(x_{i}^{+}, t\right)\right]
\end{aligned}
$$

with the convention $\dot{x}_{i}=0$ if $x_{i}=0$ or $x_{i}=x$. From these expressions, using Lemma 2, one can deduce that $w_{t}$ is wellposed for $(x, t) \in \mathscr{D}_{u}$.

Lemma 5 The infinite-dimensional backstepping transformation (32) together with the control law (6) transform the 
plant (3) into the target system

$\dot{X}(t)=(A+B K) X(t)+B w(0, t)$

$D(t) w_{t}=(1+\dot{D}(t)(x-1)) w_{x}-D(t) \dot{D}(t) g(x, t),(x, t) \in \mathscr{D}_{u}$

$w(1, t)=0$

$w\left(x, t^{+}\right)=u\left(x, t^{+}\right)-K\left[e^{A D\left(t^{+}\right) x} X(t)\right.$

$$
\left.+D\left(t^{+}\right) \int_{0}^{x} e^{A D\left(t^{+}\right)(x-y)} B u(y, t) d y\right], t \in \mathscr{T}
$$

$(\widehat{D}-D(t)) v_{t}=(1-x \dot{D}(t)) v_{x},(x, t) \in \mathscr{D}_{u}$

$v(1, t)=w(0, t)+K X(t)$

$v\left(x, t^{+}\right)=U\left(t-\underline{D}+x\left(D\left(t^{+}\right)-\widehat{D}\right)\right), t \in \mathscr{T}$

with

$$
g(x, t)=K e^{A D(t) x}(A X+B u(0, t))
$$

Proof As previously, taking time- and space-derivatives of (32), one gets, using integration by parts for the second equation,

$$
\begin{aligned}
& w_{t}=u_{t}-K \dot{D}(t)\left[e^{A D(t) x} A x X(t)\right. \\
& \left.+\int_{0}^{x} e^{A D(t)(x-y)}(I+A D(t)(x-y)) B u(y, t) d y\right] \\
& -K\left[e^{A D(t) x}(A X+B u(0, t))+D(t) \int_{0}^{x} e^{A D(t)(x-y)} B u_{t}(y, t) d y\right] \\
& -K D(t) \sum_{x_{i} \in \mathscr{X}_{(0, x)}(t)} \dot{x}_{i} e^{A D(t)\left(x-x_{i}(t)\right)} B\left(u\left(x_{i}^{-}, t\right)-u\left(x_{i}^{+}, t\right)\right) \\
& w_{x}=u_{x}-K\left[e^{A D(t) x} A D(t) X+D(t) B u(x, t)\right. \\
& +D(t) \int_{0}^{x} e^{A D(t)(x-y)} B u_{x}(y, t) d y \\
& -D(t) \\
& \quad \sum_{\left(x_{i}, x_{i+1}\right) \in \mathscr{X}_{[0, x]}(t)^{2}, x_{i}<x_{i+1}}\left[e^{A D(t)\left(x-x_{i+1}(t)\right)} B u\left(x_{i+1}^{-}, t\right)\right. \\
& \left.\left.-e^{A D(t)\left(x-x_{i}(t)\right)} B u\left(x_{i}^{+}, t\right)\right]\right]
\end{aligned}
$$

in which we have introduced

$$
\mathscr{X}_{(a, b)}(t)=\left\{x_{i} \in \mathscr{X}(t) \mid x_{i} \in(a, b)\right\},(a, b) \in[0,1]^{2}
$$

Matching those two expressions and using (22) and (26), one obtains (38) with

$$
\begin{aligned}
g(x, t) & =K\left[e^{A D(t) x} A x X(t)+(1-x) e^{A D(t) x}(A X+B u(0, t))\right. \\
+ & \left.\int_{0}^{x} e^{A D(t)(x-y)}(I+A D(t)(x-y)) B u(y, t) d y\right] \\
+ & K \int_{0}^{x} e^{A D(t)(x-y)} B(y-x) u_{x}(y, t) d y \\
+ & K \sum_{x_{i} \in \mathscr{X}(0, x)(t)}\left(x-x_{i}\right) e^{A D(t)\left(x-x_{i}\right)} B\left[u\left(x_{i}^{-}, t\right)-u\left(x_{i}^{+}, t\right)\right]
\end{aligned}
$$

which, using the following integration by parts

$$
\begin{aligned}
& \int_{0}^{x} e^{A D(t)(x-y)} B(x-y) u_{x} d y=-x e^{A D x} B u(0, t) \\
& \sum_{x_{i} \in \mathscr{X}(0, x)(t)}\left(x-x_{i}\right) e^{A D(t)\left(x-x_{i}\right)} B\left[u\left(x_{i}^{-}, t\right)-u\left(x_{i}^{+}, t\right)\right] \\
&+\int_{0}^{x} e^{A D(x-y)}(I+A D(t)(x-y)) B u(y, t) d y
\end{aligned}
$$

can be expressed as (44). The boundary condition (39) directly follows from the choice of the control law (6) and the backstepping transformation definition (32). Finally, the boundary condition (42) follows from (30) and the backstepping transformation (32) for $x=0$.

As the target system presents the suitable boundary condition $w(1, t)=0$, this is the one which is used in the Lyapunov analysis.

\subsection{Stability analysis - Proof of Theorem 1}

Consider the following Lyapunov functional candidate

$$
\begin{aligned}
V(t)= & X(t)^{T} P X(t)+b_{1} D(t) \int_{0}^{1}(1+x) w(x, t)^{2} d x \\
& +b_{2}(\widehat{D}-D(t)) \int_{0}^{1}(1+x) v(x, t)^{2} d x
\end{aligned}
$$

in which $P$ is the symmetric positive-definite solution of the Lyapunov equation $P(A+B K)+(A+B K)^{T} P=-Q$, for a given symmetric definite-positive matrix $Q$ and $b_{1}, b_{2}$ are positive constant parameters. As $D(t)$ is piecewise continuously differentiable and according to Lemma 4 , it is worth observing that this functional is piecewise continuously differentiable.

Define

$$
\Gamma_{0}(t)=|X(t)|^{2}+\int_{t-\widehat{D}}^{t} U(s)^{2} d s
$$

Note that, using Young and Cauchy-Schwarz inequalities, 
together with the inverse backstepping transformation

$$
\begin{aligned}
u(x, t)= & w(x, t)+K\left[e^{(A+B K) D(t)} X(t)\right. \\
& \left.+D(t) \int_{0}^{x} e^{(A+B K) D(t)(x-y)} B w(y, t) d y\right]
\end{aligned}
$$

one obtains the existence of constants $r_{1}, r_{2}, s_{1}, s_{2}>0$ such that

$$
\begin{aligned}
\|u(t)\|^{2} & \leq r_{1}|X(t)|^{2}+r_{2}\|w(t)\|^{2} \\
\|w(t)\|^{2} & \leq s_{1}|X(t)|^{2}+s_{2}\|u(t)\|^{2}
\end{aligned}
$$

and hence, observing that $\int_{t-D(t)}^{t} U(s)^{2} d s=D(t)\|u(t)\|^{2}$ and that $\int_{t-\widehat{D}}^{t-D(t)} U(s)^{2} d s=(\widehat{D}-D(t))\|v(t)\|^{2}$, we deduce that there are $\mu_{1}, \mu_{2}>0$ such that

$$
\mu_{1} \Gamma_{0}(t) \leq V(t) \leq \mu_{2} \Gamma_{0}(t)
$$

Now, consider $t_{i} \in \mathscr{T}$. Taking a time-derivative of (50) for $t \in\left(t_{i}, t_{i+1}\right)$ and using integrations by parts jointly with (22)(23) and Lemma 4, one gets

$$
\begin{aligned}
& \dot{V}(t)=-X^{T} Q X+2 X^{T} P B w(0, t)+b_{1}\left(-(1-\dot{D}(t)) w(0, t)^{2}\right. \\
& -\|w(t)\|^{2}-2 \dot{D}(t) \int_{0}^{1} x w(x, t)^{2} d x+\sum_{x_{i} \in \mathscr{X}_{(0,1)}(t)}\left(1+x_{i}(t)\right) \\
& \times \underbrace{\left[D(t) \dot{x}_{i}(t)+1+\dot{D}(t)\left(x_{i}(t)-1\right)\right]}_{=0}\left[w\left(x_{i}^{-}\right)^{2}-w\left(x_{i}^{+}\right)^{2}\right]) \\
& \left.-2 D(t) \dot{D}(t) \int_{0}^{1}(1+x) w(x, t) g(x, t) d x\right) \\
& +\dot{D}(t) \int_{0}^{1}(1+x)\left[b_{1} w(x, t)^{2}-b_{2} v(x, t)^{2}\right] d x \\
& +b_{2}\left(2 v(1, t)^{2}-v(0, t)^{2}-\|v(t)\|^{2}-2 \dot{D}(t) v(1, t)^{2}\right. \\
& +\dot{D}(t) \int_{0}^{1}(1+2 x) v(x, t)^{2} d x+\sum_{x_{i} \in \mathscr{X}_{(0,1)}(t)}\left(1+x_{i}(t)\right) \\
& \times \underbrace{\left[\dot{x}_{i}(t)(\widehat{D}-D(t))+1-x_{i} \dot{D}(t)\right]}_{=0}\left[v\left(x_{i}^{-}, t\right)^{2}-v\left(x_{i}^{+}, t\right)^{2}\right])
\end{aligned}
$$

in which, from (30) and (32),

$$
2 v(1, t)^{2} \leq 4\left(w(0, t)^{2}+|K|^{2}|X(t)|^{2}\right)
$$

Using the fact that, from (6) with Young and Cauchy-
Schwarz inequalities,

$$
\begin{aligned}
u(0, t)^{2} & =U(t-D(t))^{2} \\
& \leq \tilde{M}\left(|X(t-D(t))|^{2}+\|u(t-D(t))\|^{2}\right), t \geq \bar{D}
\end{aligned}
$$

for a given positive constant $\tilde{M}$, together with (53) and Young and Cauchy-Schwarz inequalities, one obtains the existence of a constant $M>0$ such that

$$
\begin{aligned}
& \left|2 D(t) \int_{0}^{1}(1+x) w(x, t) g(x, t) d x\right| \\
& \leq M\left(\max _{s \in[-\bar{D}, 0]}|X(t+s)|^{2}+\max _{s \in[-\bar{D}, 0]}\|w(t+s)\|^{2}\right)
\end{aligned}
$$

$$
\begin{aligned}
& 2 v(1, t)^{2} \leq M\left(\max _{s \in[-\bar{D}, 0]}|X(t+s)|^{2}+\max _{s \in[-\bar{D}, 0]}\|w(t+s)\|^{2}\right) \\
& w(0, t)^{2} \leq M\left(\max _{s \in[-\bar{D}, 0]}|X(t+s)|^{2}+\max _{s \in[-\bar{D}, 0]}\|w(t+s)\|^{2}\right)
\end{aligned}
$$

for $t \geq \bar{D}$. Therefore, with (57), (59)-(61) and applying Young inequality, one gets f.a.a. $t \geq \bar{D}$

$$
\begin{gathered}
\dot{V}(t) \leq-\left(\frac{\underline{\lambda}(Q)}{2}-4 b_{2}|K|^{2}\right)|X(t)|^{2}-b_{1}\|w(t)\|^{2} \\
-b_{2}\|v(t)\|^{2}-\left(b_{1}-4 b_{2}-\frac{2|P B|^{2}}{\underline{\lambda}(Q)}\right) w(0, t)^{2} \\
+b_{0}|\dot{D}(t)|\left(\max _{s \in[-\bar{D}, 0]}|X(t+s)|^{2}+\max _{s \in[-\bar{D}, 0]}\|w(t+s)\|^{2}\right)
\end{gathered}
$$

in which $b_{0}=b_{1}(4+2 M)+b_{2}(5+M)$. Consequently, choosing $b_{2}=\frac{\lambda(Q)}{16|K|^{2}}, b_{1}>4 b_{2}+\frac{2|P B|^{2}}{\underline{\lambda}(Q)}$, it follows

$$
\dot{V}(t) \leq-\eta V(t)+\eta_{0}|\dot{D}(t)| \max _{s \in[-\underline{D}, 0]} V(t+s), \text { f.a.a. } t \geq \bar{D}
$$

in which we have introduced $\eta=\frac{\min \left\{\frac{\lambda(Q)}{4}, b_{2}\right\}}{\max \left\{\bar{\lambda}(P), 2 b_{1} \widehat{D}\right\}}$ and $\eta_{0}=\frac{b_{0}}{\min \left\{\underline{\lambda}(P), b_{1} \underline{D}\right\}}$. We now consider (63) for $t \in\left(t_{i}, t_{i+1}\right)$ and introduce $W$ such that

$$
\begin{aligned}
& \dot{W}(t)=-\eta W(t)+b(t) \max _{s \in[-\underline{D}, 0]} W(t+s), \text { f.a.a. } t \in\left(t_{i}, t_{i+1}\right] \\
& W(t)= \begin{cases}V(t) & \text { if } t \in\left[t_{i}-\bar{D}, t_{i}\right) \\
\max \left\{V\left(t_{i}^{+}\right), V\left(t_{i}^{-}\right)\right\} & \text {if } t=t_{i}\end{cases}
\end{aligned}
$$


in which $b$ is a function such that $b(t)=\eta_{0}|\dot{D}(t)|$ for $t \in$ $\left(t_{i}, t_{i+1}\right)$. Such a solution is well-defined, as Assumption 1(ii) guarantees that the initial condition is piecewise continuous and according to Lemma 7. Applying Lemma 6, one concludes that there exists $\delta^{*} \in\left(0,\left(\frac{\eta}{\eta_{0}}\right)^{2}\right)$ such that, for $\delta<\delta^{*}$, there exist two constants $r, \gamma>0$ (independent of $t_{i+1}-t_{i}$ ) such that, for $t \geq \bar{D}$,

$$
W(t) \leq r \max _{s \in[-\bar{D}, 0]} W\left(t_{i}+s\right) e^{-\gamma\left(t-t_{i}\right)}, t \in\left[t_{i}, t_{i+1}\right)
$$

Considering $z=W-V$, with a contradiction argument (similarly to the one employed in the proof of Lemma 6), one can conclude that $z(t) \geq 0$ for $t \in\left[t_{i}, t_{i+1}\right)$ and thus that

$$
V(t) \leq r \max _{s \in[-\bar{D}, 0]} W\left(t_{i}+s\right) e^{-\gamma\left(t-t_{i}\right)}, t \in\left[t_{i}, t_{i+1}\right)
$$

Hence, for $\delta<\delta^{*}$, using (55), the definition (65) and the fact that $\Gamma_{0}$ is continuous, one deduces the existence of $\tilde{R}, \tilde{\gamma}>0$ such that, for $t \geq \bar{D}$,

$$
\Gamma_{0}(t) \leq \tilde{R} \max _{s \in[-\bar{D}, 0]} \Gamma_{0}\left(t_{i}+s\right) e^{-\tilde{\gamma}\left(t-\max \left\{t_{i}, \bar{D}\right\}\right)}, t \in\left[t_{i}, t_{i+1}\right)
$$

Consequently, as $\Gamma$ is a continuous functional, one gets

$$
\max _{s \in[-\bar{D}, 0]} \Gamma_{0}\left(t_{i+1}+s\right) \leq \tilde{R} e^{-\tilde{\gamma}\left(\Delta t_{i}-\bar{D}\right)} \max _{s \in[-\bar{D}, 0]} \Gamma_{0}\left(t_{i}+s\right)
$$

in which, potentially, $\Delta t_{i} \leq \bar{D}$. Hence, with $N_{D}(t, \tau)$ the number of discontinuities in the interval $(\tau, t)$, it follows that

$$
\Gamma_{0}(t) \leq \tilde{R}^{N_{D}(t, \bar{D})} e^{-\tilde{\gamma}\left(t-\bar{D}-N_{D}(t, \bar{D}) \bar{D}\right)} \max _{s \in[-\bar{D}, 0]} \Gamma_{0}(\bar{D}+s)
$$

or equivalently, using (9)

$$
\Gamma_{0}(t) \leq e^{N_{0}(\ln (\tilde{R})+\tilde{\gamma} \bar{D})} e^{-\left(\tilde{\gamma}-\frac{\ln (\tilde{R})+\tilde{\gamma} \bar{D}}{\tau_{D}}\right)(t-\bar{D})} \max _{s \in[-\bar{D}, 0]} \Gamma_{0}(\bar{D}+s)
$$

Consequently, if $\tau_{D}>\tau_{D}^{*} \triangleq \frac{\ln (\tilde{R})}{\tilde{\gamma}}+\bar{D}$ and as $\widehat{D}$ can be chosen arbitrarily close to $\bar{D}$, there exist two constants $R, \gamma>0$ such that the exponential stability result in terms of $\Gamma$ of Theorem 1 holds.

\section{Illustrative toy example: communication delay}

To illustrate the relevance of the proposed prediction-based control law, we consider the unstable second-order dynamics

$$
\dot{X}(t)=\left(\begin{array}{cc}
0 & 1 \\
-1 & 1
\end{array}\right) X(t)+\left(\begin{array}{l}
0 \\
1
\end{array}\right) U(t-D(t))
$$

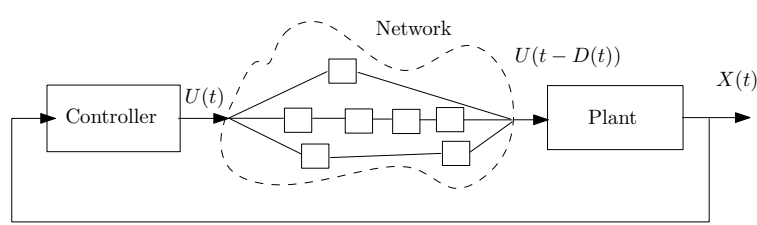

Fig. 3. Schematic view of the considered system with communication delay. The controller and the plant exchange information through a network in which a supervisor orchestrates the data routing by choosing between a family of candidates paths.

in which $D(t)$ is a piecewise continuously differentiable delay function as considered throughout this paper. It can be subject to large variations. A schematic view of the system at stake is given in Fig. 3. We consider that the controller sends orders to the plant through a network in which a supervisor orchestrates the data routing by choosing between a family of candidate paths. This routing tries to keep the data queuing lines below some acceptable value. If it increases too much, a new route is chosen, causing delay jumps. We assume that the communication channels between the plant and the controller are not symmetric, resulting into a sole input delay ${ }^{2}$.

We first consider the delay function pictured in Fig. 4(b). In this case, data were routed in such a way that reordering occurs periodically, resulting in periodic jumps and non-FIFO delay variations ( $\dot{D}$ exhibits values larger than one periodically). The control law (6) is applied with the feedback gain $K=-\left[\begin{array}{ll}9 & 10\end{array}\right]$ and implemented with a trapezoidal discretization of the integral. Closed-loop simulation results are reported in Fig. 4. One can observe that the plant asymptotically converges, as Theorem 1 guarantees it can be the case. Indeed, the intervals during which the delay derivative is larger than one are reduced enough compared to the dwell time (constant in this example) to guarantee that the condition $\delta<\delta^{*}$ required by Theorem 1 holds. On the other hand, a similar controller but using a prediction based on a constant average delay value $(D \approx 0.22)$ fails to stabilize the plant. In all likelihood, this result could be explained by the selection of a relatively high feedback gain value $K=-[910]$. This illustrates the interest of using the current delay value as prediction horizon rather than an average value of it, grounding in a delay-robustness property of the prediction-based controller (as studied in a FIFO context in [6]).

To evaluate our controller performance in a more challenging context, we consider now that the delay is a discrete-time random process, $(D(n))_{n \in \mathbb{N}}=\left(D\left(n T_{s}\right)\right)_{n \in \mathbb{N}}$ with $T_{s}=0.3 \mathrm{~s}$ and $D(n)$ a uniform random variable on $[0.8,1.2]$. The control law (6) is now applied with the feedback gain $K=$ - $\left[\begin{array}{ll}3 & 4\end{array}\right]$. Corresponding simulation results are pictured in

\footnotetext{
2 An even more general and representative modeling could also include a time-varying output delay (to account for the fact that the plant sends outputs to the controller through a similar network). To handle this additive complexity, our control strategy should be extended to handle linear time-varying dynamics.
} 

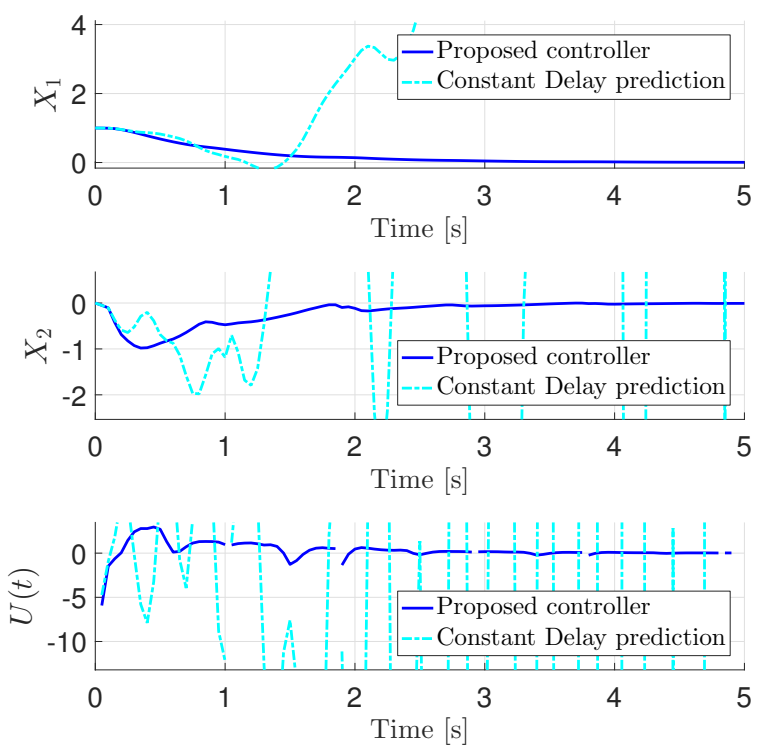

(a) State and control evolution.
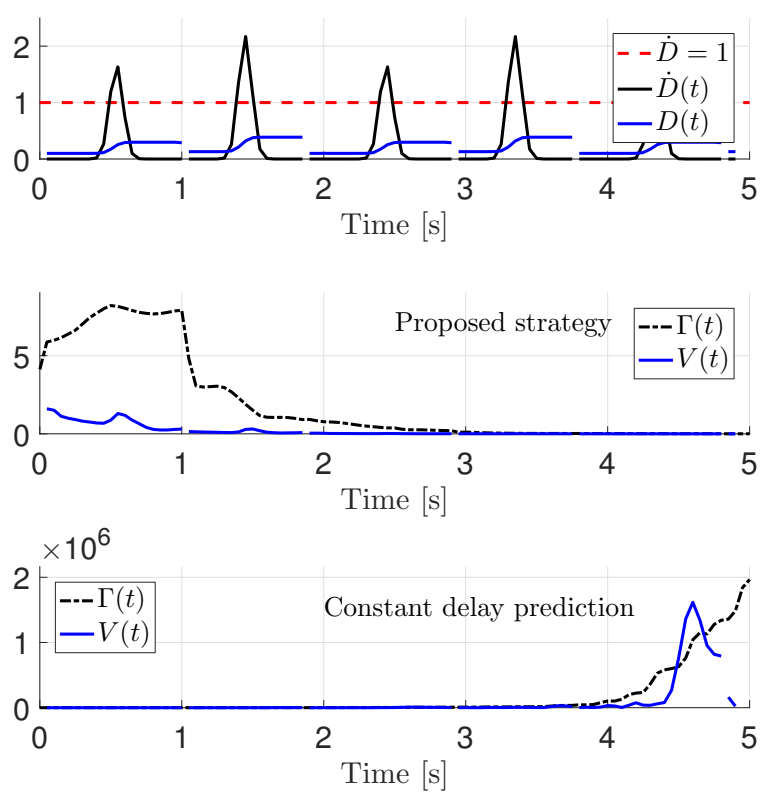

(b) Delay and functionals evolution.

Fig. 4. Simulation results with a feedback gain $K=-\left[\begin{array}{ll}9 & 10\end{array}\right]$ and initial conditions $X(0)=\left[\begin{array}{ll}1 & 0\end{array}\right]^{T}, U_{0}=0$. The controller proposed in this paper is compared with a prediction-based controller using a constant delay $D \approx 0.22$.

Fig. 5 and exhibit the same convergence property as previously. Similarly, a prediction-based controller using the expected delay value $E(D(n))=1$ as prediction horizon fails to stabilize the plant. Of course, this framework does not fit into the mathematical formalism considered throughout this paper, which could be considered as a first step to address this case. This is a direction of future works, the interest of which is strengthen by the simulation results of Fig. 5 .
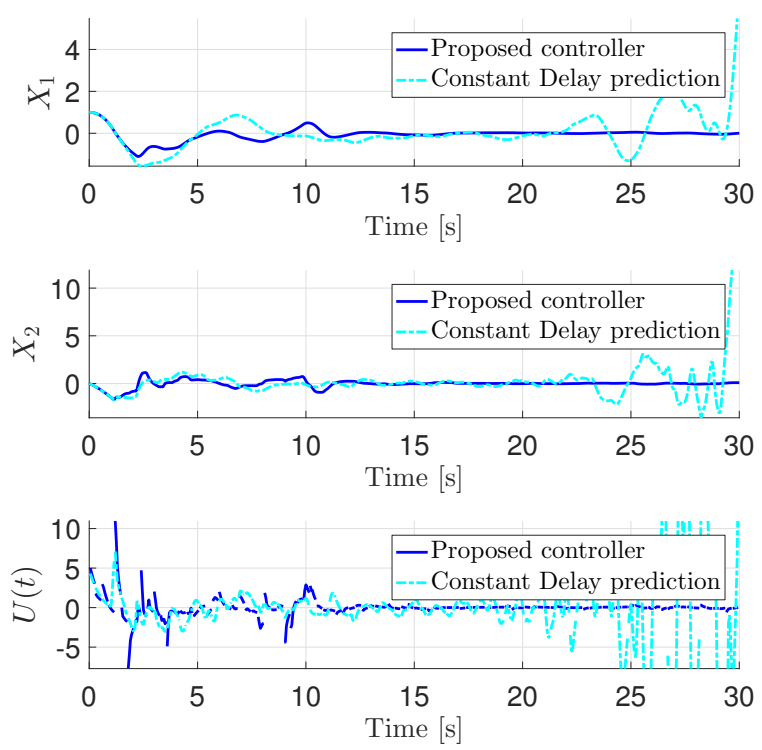

(a) State and control evolution.
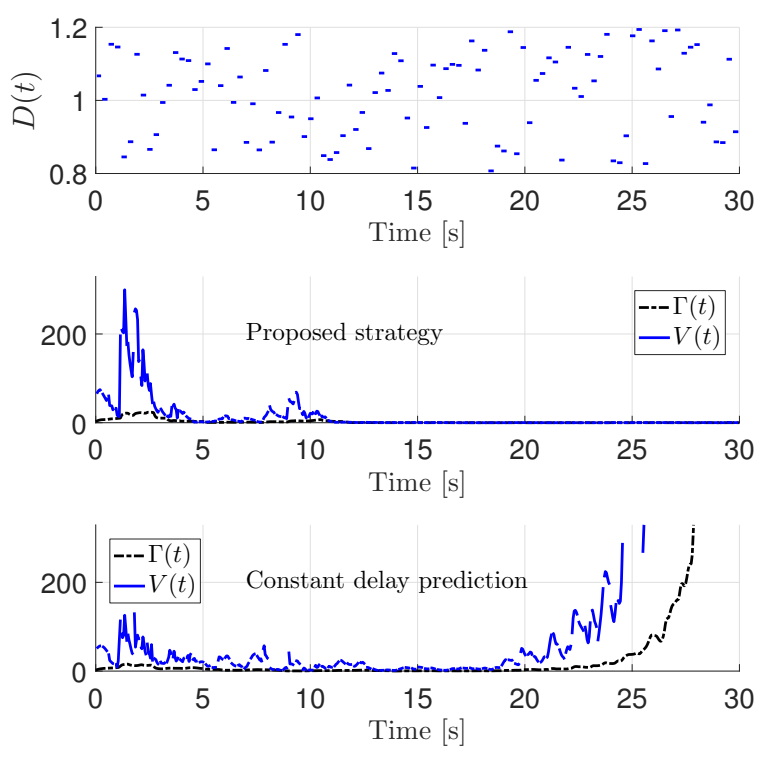

(b) Delay and functionals evolution.

Fig. 5. Simulation results with a feedback gain $K=-\left[\begin{array}{ll}3 & 4\end{array}\right]$ and initial conditions $X(0)=\left[\begin{array}{ll}1 & 0\end{array}\right]^{T}, U_{0}=0$. The delay is a discretetime random process, $(D(n))_{n \in \mathbb{N}}=\left(D\left(n T_{s}\right)\right)_{n \in \mathbb{N}}$ with $T_{s}=0.3 \mathrm{~s}$ and $D(n)$ a uniform random variable on $[0.8,1.2]$. The controller proposed in this paper is compared with a prediction-based controller using a constant delay $D=1$.

\section{Conclusion}

This paper presents a prediction-based control for a timevarying input delay, the variations of which are not assumed to satisfy a FIFO property and are not assumed to be continuous or with sufficiently small jumps. We propose to use the current delay as a prediction horizon and proved that the closed-loop system exponentially converges, provided that the delay time-derivative is sufficiently small in the sense of 
an average $\mathscr{L}_{2}$-norm and that the delay discontinuities are sufficiently sparse in the sense of the average dwell time. This result is very promising as it enables to alleviate the very limiting assumption $\dot{D}(t)<1, t \geq 0$ and to consider delays with strong discontinuities.

Much more remains to be done. Extension of this work to more general dynamics, such as time-varying or nonlinear ones, is a first path to explore. This will likely require the design of new Halanay-type Delay Differential Inequalities. Further, the interest of Lyapunov techniques standardly used in the field of sampled-data systems (looped functionals) should be investigated in this context.

Appendix A: Well-posedness of (26)-(31) - Proof of Lemma 3

We start this proof by noticing that (11)-(12) is a solution to the system (26)-(31). We now wish to prove that this solution is unique and depends continuously on its initial condition. With this aim in view, consider $(u, v)$ a solution to (26)-(31) and introduce the following distributed variable

$$
\bar{u}(x, t)= \begin{cases}u\left(\frac{\bar{D}}{D(t)}(x-1)+1, t\right), & x \in\left[\frac{\bar{D}-D(t)}{\bar{D}}, 1\right] \\ v\left(\frac{\bar{D}}{\bar{D}-D(t)} x, t\right), & x \in\left[0, \frac{\bar{D}-D(t)}{\bar{D}}\right]\end{cases}
$$

which can be inverted as

$$
\left\{\begin{array}{l}
u(x, t)=\bar{u}\left(1+\frac{D(t)}{\bar{D}}(x-1), t\right) \\
v(x, t)=\bar{u}\left(\frac{\bar{D}-D(t)}{\bar{D}} x, t\right)
\end{array}\right.
$$

Note that the variable (70) is well-defined as $\bar{u}\left(\frac{\bar{D}-D(t)}{\bar{D}}\right)=$ $u(0, t)=v(1, t)$ according to (30). Furthermore, taking timeand space-derivatives of $\bar{u}$ and using (26)-(31), one proves that it satisfies

$$
\begin{aligned}
\bar{D} \bar{u}_{t} & =\bar{u}_{x} \\
\bar{u}(1, t) & =U(t)
\end{aligned}
$$

which has a unique weak solution in $L_{2}(0,1)$ (see [11]). Thus, from its inverse (71), this implies that $(u, v)$ is the unique weak solution of (26)-(31) and depends continuously on its initial condition.

\section{Appendix B: Time-varying Halanay inequality}

We use the following result, the proof of which is inspired from [22].
Lemma 6 Consider a nonnegative piecewise continuously differentiable function $x$ with only one discontinuity at time $t=0$ and such that

$$
\left\{\begin{aligned}
\dot{x}(t) & \leq-a x(t)+b(t) \max _{s \in[-\bar{D}, 0]} x(t+s), \quad \text { f.a.a. } t>0 \\
x_{0} & =\psi \in \mathscr{C}_{p w}([-\bar{D}, 0], \mathbb{R})
\end{aligned}\right.
$$

in which $\bar{D} \geq 0, a \geq 0$ and $b: \mathbb{R}_{+} \rightarrow \mathbb{R}_{+}$is a piecewise continuous function with only one discontinuity at time $t=0$ and which satisfies for some $T>0, \delta>0$ and $T_{0}>\bar{D}+T$

$$
\frac{1}{T} \int_{t}^{t+T} b(s)^{2} d s \leq \delta, t \in\left[0, T_{0}-T\right]
$$

There exists $\delta^{*} \in\left(0, a^{2}\right)$ (independent of $\left.T_{0}\right)$ such that, if $\delta<\delta^{*}$, then there exist two constants $\gamma, r \geq 0$ (independent of $T_{0}$ ) such that

$$
\forall t \geq t_{0} \quad x(t) \leq r \max \psi e^{-\gamma t}, \quad t \in\left[0, T_{0}\right]
$$

Proof We start by proving the existence of $r_{0}>0$ such that

$$
\max _{s \in[-T-\bar{D}, 0]} x(T+s) \leq r_{0} \max \psi
$$

Let $k>\max \psi$ and $y$ such that $y(t)=k$ for $t \in[-\bar{D}, 0]$ and $y(t)=k \exp \left(\int_{0}^{t} b(s) d s\right)$ for $t \in[0, T]$. Thus, $y$ is an increasing function which satisfies

$$
\dot{y}(t)=b(t) \max _{s \in[-\bar{D}, 0]} y(t+s), \quad t>0
$$

Consider $z=y-x$ and, by contradiction, assume there exists $t_{1}$ such that $z\left(t_{1}\right)<0$. Then, by continuity, there exists $t_{2}$ such that

$$
\begin{aligned}
& z(t)>0 \text { for } t \in\left[0, t_{2}\right) \\
& z\left(t_{2}\right)=0 \\
& \dot{z}\left(t_{2}\right) \leq 0
\end{aligned}
$$

However, from (74) and (78), it follows that

$$
\dot{z}\left(t_{2}\right) \geq b\left(t_{2}\right) y\left(t_{2}\right)-b\left(t_{2}\right) \max x\left(t_{2}\right)>0
$$

in which the last inequality follows from the definition of $t_{2}$ and is in contradiction with (79). Therefore, $z(t) \geq 0, t \in$ $[0, T]$. As the previous considerations hold for all $k>\max \psi$, using Cauchy-Schwarz inequality, one concludes that

$$
\begin{aligned}
x(t) & \leq \exp \left(\int_{0}^{t} b(s) d s\right) \max \psi \\
& \leq \exp \left(\sqrt{\int_{0}^{t} b(s)^{2} d s \sqrt{t}}\right) \max \psi, \quad t \in[0, T]
\end{aligned}
$$


As $t \in[0, T]$, using (75), it follows that (77) holds with $r_{0}=e^{\sqrt{\delta T} \sqrt{T}}$.

Now, consider $t \geq T$. Integrating (74) between $t-T$ and $t$, one gets

$$
\begin{aligned}
x(t) & \leq e^{-a T} x(t-T)+\int_{t-T}^{t} e^{-a(t-s)} b(s) \max _{\xi \in[-\bar{D}, 0]} x(s+\xi) d s \\
& \leq\left(e^{-a T}+\int_{t-T}^{t} e^{-a(t-s)} b(s) d s\right) \max _{s \in[-T-\bar{D}, 0]} x(t+s)
\end{aligned}
$$

in which the right-hand side is well-defined according to Lemma 7. Using Cauchy-Schwarz inequality and from (75), one obtains

$$
\begin{aligned}
x(t) & \leq\left(e^{-a T}+\sqrt{\frac{1-e^{-2 a T}}{2 a}} \sqrt{\int_{t-T}^{t} b(s)^{2} d s}\right) \max _{s \in[-T-\bar{D}, 0]} x(t+s) \\
& \leq c \max _{s \in[-T-\bar{D}, 0]} x(t+s)
\end{aligned}
$$

in which

$$
c=e^{-a T}+\sqrt{\frac{1-e^{-2 a T}}{2 a}} \sqrt{T \delta}
$$

Thus, with (77), if $c<1$, the result holds. With straightforward calculations, one obtains that $c<1$ if and only if

$$
\delta \leq \frac{2 a}{T} \frac{1-e^{-a T}}{1+e^{a T}} \triangleq \delta^{*}
$$

Finally, one can note that $\delta^{*}$ is a decreasing function with respect to $T$ which tends to $a^{2}$ as $T$ tends to zero. This gives the expected result.

In the above, we also needed the following result.

Lemma 7 Consider $f \in \mathscr{C}_{p w}(\mathbb{R}, \mathbb{R})$ and $\bar{D} \geq 0$, then

$$
h: t \in \mathbb{R} \mapsto \max _{s \in[t-\bar{D}, t]} f(s) \in \mathscr{C}_{p w}(\mathbb{R}, \mathbb{R})
$$

Proof The main idea of this proof is to construct a new grid for $h$ which gathers the grid corresponding to $f$ and the same one but delayed by $D$-units of time.

Consider $I=[a, b]$. Define $\tilde{I}=[a-\bar{D}, b]$ and a finite sequence $\left(\tilde{t}_{n}\right)_{0 \leq n \leq \tilde{N}}$ partitioning $\tilde{I}$ and corresponding to the piecewise continuous function $f$. Define

$$
t_{0}=a, t_{i+1}=\min \left\{\min _{\substack{0 \leq n \leq \tilde{N} \\ t_{i}<\tilde{t}_{n}}} \tilde{t}_{n}, \bar{D}+\min _{\substack{0 \leq n \leq \tilde{N} \\ t_{i}-\bar{D}<\tilde{t}_{n}}} \tilde{t}_{n}\right\}
$$

By construction, this sequence is finite and $a=t_{0}<t_{1}<$ $\ldots<t_{N}=b$ (for a given $N \in \mathbb{N}$ ). Consider $t \in\left(t_{i}, t_{i+1}\right)$. By definition, as $[t-\bar{D}, t]=\left[t-\bar{D}, t_{i+1}-\bar{D}\right) \cup\left[t_{i+1}-\bar{D}, t_{i}\right] \cup$ $\left(t_{i}, t\right]$

$h(t)=\max \left\{\sup _{s \in\left[t-\bar{D}, t_{i+1}-\bar{D}\right)} f(s), \max _{s \in\left[t_{i+1}-\bar{D}, t_{i}\right]} f(s), \sup _{s \in\left(t_{i}, t\right]} f(s)\right\}$

or, denoting $f^{e}$ the continuity extension of $f$ (which exists as $f$ is piecewise continuous),

$$
h(t)=\max \left\{\max _{s \in\left[t-\bar{D}, t_{i+1}-\bar{D}\right]} f^{e}(s), \max _{s \in\left[t_{i+1}-\bar{D}, t_{i}\right]} f(s), \max _{s \in\left[t_{i}, t\right]} f^{e}(s)\right\}
$$

Finally, applying the maximum theorem [7], one concludes that $t \mapsto \max _{s \in\left[t-\bar{D}, t_{i+1}-\bar{D}\right]} f^{e}(s)$ and $t \mapsto \max _{s \in\left[t_{i}, t\right]} f^{e}(s)$ are continuous functions as $f^{e}$ is continuous over the intervals under consideration. Therefore, it follows that $h$ is continuous over $\left(t_{i}, t_{i+1}\right)$ by composition of continuous functions. One also concludes that $h$ admits a finite left-hand side limit at $t_{i+1}$ and right-hand site limit at $t_{i}$, which concludes the proof.

\section{References}

[1] Z. Artstein. Linear systems with delayed controls: a reduction. IEEE Transactions on Automatic Control, 27(4):869-879, 1982.

[2] N. Bekiaris-Liberis and M. Krstic. Compensation of time-varying input and state delays for nonlinear systems. Journal of Dynamic Systems, Measurement and Control, 134, 2012. Paper 011009.

[3] N. Bekiaris-Liberis and M. Krstic. Compensation of state-dependent input delay for nonlinear systems. IEEE Transactions on Automatic Control, 58:275- 289, 2013.

[4] N. Bekiaris-Liberis and M. Krstic. Nonlinear control under delays that depend on delayed states. European Journal on Control, Special Issue for the ECC13, 19:389-398, 2013.

[5] N. Bekiaris-Liberis and M. Krstic. Nonlinear Control Under Nonconstant Delays, volume 25. Society for Industrial and Applied Mathematics, 2013.

[6] N. Bekiaris-Liberis and M. Krstic. Robustness of nonlinear predictor feedback laws to time-and state-dependent delay perturbations. Automatica, 49:1576-1590, 2013.

[7] C. Berge. Topological Spaces: including a treatment of multi-valued functions, vector spaces, and convexity. Courier Corporation, 1963.

[8] D. Bresch-Pietri, J. Chauvin, and N. Petit. Adaptive control scheme for uncertain time-delay systems. Automatica, 48:1536-1552, 2012.

[9] D. Bresch-Pietri and N Petit. Robust compensation of a chattering time-varying input delay. In Proc. of the Conference on Decision and Control, 2014.

[10] M. Chèbre, Y. Creff, and N. Petit. Feedback control and optimization for the production of commercial fuels by blending. Journal of Process Control, 20(4):441-451, 2010.

[11] Ruth F Curtain and Hans Zwart. An introduction to infinitedimensional linear systems theory. 1995.

[12] C. Depcik and D. Assanis. One-dimensional automotive catalyst modeling. Progress in energy and combustion science, 31(4):308369, 2005. 
[13] J.-Y. Dieulot and J.-P. Richard. Tracking control of a nonlinear system with input-dependent delay. Proc. of the 40th Conference on Decision and Control, 4:4027-4031, 2001.

[14] L. Figueredo, J. Y. Ishihara, Borges G. A., and A. Bauchspiess. Robust stability criteria for uncertain systems with delay and its derivative varying within intervals. In American Control Conference, 2011.

[15] K. Gu and S. I. Niculescu. Survey on recent results in the stability and control of time-delay systems. Journal of Dynamic Systems, Measurement, and Control, 125:158, 2003.

[16] J. P. Hespanha and S. Morse. Stability of switched systems with average dwell-time. In Proceedings of the 38th IEEE Conference on Decision and Control, volume 3, pages 2655-2660. IEEE, 1999.

[17] M. Jankovic. Recursive predictor design for linear systems with time delay. In American Control Conference, pages 4904-4909, 2008.

[18] M. Krstic. Delay Compensation for Nonlinear, Adaptive, and PDE Systems. Birkhauser, 2009.

[19] M. Krstic and A. Smyshlyaev. Boundary Control of PDEs: a Course on Backstepping Designs. Society for Industrial and Applied Mathematics Philadelphia, PA, USA, 2008.

[20] W. Kwon and A. Pearson. Feedback stabilization of linear systems with delayed control. IEEE Transactions on Automatic Control, 25(2):266-269, 1980.

[21] A. Manitius and A. Olbrot. Finite spectrum assignment problem for systems with delays. IEEE Transactions on Automatic Control, 24(4):541-552, 1979.

[22] F. Mazenc and M. Malisoff. Trajectory based approach for the stability analysis of nonlinear systems with time delays. IEEE Transactions on Automatic Control, 60(6), June 2015.

[23] F. Mazenc, M. Malisoff, and S.-I. Niculescu. Stability and control design for time-varying systems with time-varying delays using a trajectory-based approach. SIAM Journal on Control and Optimization, 55(1):533-556, 2017.

[24] F. Mazenc and S. I. Niculescu. Generating positive and stable solutions through delayed state feedback. Automatica, 47:525-533, 2011.

[25] F. Mazenc, S. I. Niculescu, and M. Krstic. Lyapunov-Krasovskii Functionals and Application to Input Delay Compensation for Linear Time-Invariant Systems. Automatica, 48:1317-1323, July 2012.

[26] W. Michiels and S. I. Niculescu. Stability and Stabilization of TimeDelay Systems. Society for Industrial and Applied Mathematics, 2007.

[27] Y. S. Moon, P. G. Park, and W. H. Kwon. Robust stabilization of uncertain input-delayed systems using reduction method. Automatica, 37(2):307-312, 2001.

[28] M. T. Nihtila. Finite pole assignment for systems with time-varying input delays. In Proc. of the 30th IEEE Conference on Decision and Control, pages 927-928, 1991.

[29] N. Petit, Y. Creff, and P. Rouchon. Motion planning for two classes of nonlinear systems with delays depending on the control. In Proceedings of the 37th IEEE Conference on Decision and Control, pages 1007-1011, 1998.

[30] J.-P. Richard. Time-delay systems: an overview of some recent advances and open problems. Automatica, 39(10):1667-1694, 2003.

[31] M. Sbarciog, R. De Keyser, S. Cristea, and C. De Prada. Nonlinear Predictive Control of processes with variable time delay. A temperature control case study. In Proc. of the IEEE International Conference on Control Applications, pages 1001-1006, 2008.

[32] A. Seuret, F. Gouaisbaut, and E. Fridman. Stability of systems with fast-varying delay using improved wirtinger's inequality. In Decision and Control (CDC), 2013 IEEE 52nd Annual Conference on, pages 946-951. IEEE, 2013.
[33] E. Shustin and E. Fridman. On delay-derivative-dependent stability of systems with fast-varying delays. Automatica, 43(9):1649-1655, 2007.

[34] O. J. M. Smith. A controller to overcome dead time. ISA Journal, 6(2):28-33, 1959.

[35] E. Witrant. Stabilisation des systèmes commandés par réseaux. $\mathrm{PhD}$ thesis, Laboratoire d'Automatique de Grenoble, 2005.

[36] D. Yue and Q. L. Han. Delayed feedback control of uncertain systems with time-varying input delay. Automatica, 41(2):233-240, 2005. 